\title{
Perioperative Awareness after Anesthesia: A Case Report
}

\author{
Caroline Wangui Njue ${ }^{1}$, Ngugi M. Kinyungu ${ }^{2}$ \\ ${ }^{1}$ St George's University, Grenada \\ ${ }^{2}$ ITAV Pain Management, Donald and Barbara Zucker School of Medicine at Hofstra/Northwell, New York, USA \\ Email: cwangui13@gmail.com
}

How to cite this paper: Njue, C.W. and Kinyungu, N.M. (2021) Perioperative Awareness after Anesthesia: A Case Report. Open Journal of Anesthesiology, 11, 149-155. https://doi.org/10.4236/ojanes.2021.115014

Received: March 12, 2021

Accepted: May 9, 2021

Published: May 12, 2021

Copyright $\odot 2021$ by author(s) and Scientific Research Publishing Inc. This work is licensed under the Creative Commons Attribution International License (CC BY 4.0).

http://creativecommons.org/licenses/by/4.0/

\begin{abstract}
Background: Accidental awareness during general anesthesia (AAGA) is an uncommon condition with possible long-term sequela. Here, we identify and describe a case of awareness in the perioperative setting and the resulting sequelae of symptoms consistent with post-traumatic stress disorder using the first-hand experience of a patient with awareness during anesthesia. Aim: This case report aims to identify and describe the symptomatic sequelae of perioperative awareness events and the importance of close patient follow-up and management. Case Presentation: A 34-year-old female developed uncontrolled tonsillar hemorrhage 2 days after successful adenotonsillectomy that necessitated bedside intubation. During regular clinic follow-up, the patient presented with symptoms suggestive of post-traumatic stress disorder (PTSD) secondary to recall events while under anesthesia. Conclusion: The contributory factors predisposing to perioperative awareness in the surgical environment need to be understood and avoided as much as possible. When awareness is suspected or known to have occurred the patient needs to be assisted through the post-op period and guided through the psychological sequelae that follow.
\end{abstract}

\section{Keywords}

Accidental Awareness during General Anesthesia, Post-Traumatic Stress Disorder

\section{Introduction}

Accidental awareness during general anesthesia (AAGA) is the terminology used for the explicit recall of operative events especially sensory perception by a patient who underwent general anesthesia [1]. Although rare, the after-effects can 
be extremely agonizing for the patient and the anesthetist. Recall of conversation among the staff and feeling of excruciating pain lingers in the minds of these patients and can lead to long-term psychological issues and could ultimately pave the way to medical litigation. An analysis of the ASA Closed Claims Project by Domino et al. [2] revealed that out of 4183 claims, 61 accounted for recall during general anesthesia. Age less than 60 years (89\%) women (77\%), elective procedures (87\%), and American Society of Anesthesiologists physical class 1-1l (68\%) were factors noted. Alternative contributory factors noted include patient's age, drug resistance, pre-existing psychiatric disorder, type of surgeries where "light anesthesia" may be used, anesthetic machine malfunctioning, and suboptimal dosing of anesthetic drugs [3].

We report here a patient's case of perioperative awareness with post-hospitalization symptoms consistent with PTSD secondary to the event.

\section{Case Presentation}

A 34-year-old female presented to the Emergency Department in the middle of the covid-19 pandemic with complaints of sore throat, fever, difficulty swallowing, and hematemesis. The patient had a medical history of laparoscopic vertical sleeve gastrectomy, rheumatoid arthritis, inflammatory bowel disease, and fibromyalgia. The patient was diagnosed with tonsillitis with hemorrhage, and she underwent a successful emergency adenotonsillectomy with no complications and she was subsequently discharged.

She then came into the Emergency department two days later with dehydration, difficulty swallowing, and hematemesis. CT scan of the neck indicated a soft tissue swelling in the hypopharynx, with a possible abscess developing. She was taken urgently to the operating room for exploration and control of the tonsil bleed. Upon completion of a successful operation, she was extubated and admitted under observation. Two days later, she had a recurrence of tonsillar bleeding and was again taken to the operating room to control the bleeding after which she was extubated. A few hours after extubating, she had abrupt onset hemoptysis and was urgently intubated at her bedside and sedated. The neurological intervention team was consulted, and the patient was taken urgently for embolization of a bleeding right lingual artery.

It was during the bedside intubation that the patient experienced recall. She stated that she recalled the doctor telling her "go to sleep and you will have a breathing tube". During the period, she also recalls hearing someone say that "we might lose her". She states she lost consciousness for a brief period and when she came to, she was aware that she had a breathing tube and could hear everything but was unable to open her eyes or move. She stated she tried very hard to move any part of her body to signal that she was awake with no success. She also tried changing her breathing pattern so someone could notice but to no avail. She added that she was in a dire state of panic as the time seemed to slow down. She states eventually she heard someone say, "I think she is awake" and at 
that time her mind went "blank" and the next event she recalled was waking up in the catheterization lab after the neuro-intervention procedure. With the bleeding now controlled the patient was discharged from the hospital a few days later.

Outpatient Management

Upon discharge, the patient was seen for regular follow-up at the pain clinic whereupon she relayed severe symptoms of anxiety and distress due to recall from the event. The patient said that she had a hard time going to bed and getting regular sleep for weeks after discharge. She stated she was unable to sleep for more than an hour or two at a time for weeks after the event. She was very stressed and anxious during the day. Multiple times she has had flashbacks of the event in which she could recall the event vividly. She reports having dismal dreams and nightmares. Panic attacks during the day were quite common. The patient stated she could not face a situation with confidence and a sense of impending doom loomed over her always and she felt claustrophobic at times. She tried to avoid recalling this peri-operative awareness experience with no success.

The patient had not relayed the events to any other provider by the time she relayed the symptoms during her follow-up visit with the pain clinic. The events of awareness under anesthesia were discussed with her at the pain clinic and she was educated and consoled with an extensive visit. She was advised to seek help from a mental health specialist however due to the Covid 19 lockdown she was unable to find a practitioner even after she tried multiple times. She continued to receive close guidance from the pain clinic and through this guidance, after a couple of months, she was able to function better and sleep well with reduced anxiety.

The management of the post-awareness symptoms in the outpatient setting included close follow-up by her provider including biweekly discussions via telehealth in the immediate period following when her symptoms were severe. During this discussion, the patient was given a chance to relay her feelings and experiences during the episode with an affirmation of what she went through. The patent expressed relief at being able to talk about the episodes as she felt no one else around her understood what she went through and this made it even more frustrating and anxiety-provoking as she felt that maybe it was "all in her head". The patient was also managed with a low dose benzodiazepine to help with the anxiety especially at night and this was tapered off and stopped in three months after her symptoms had lessened and she was able to function better.

Follow-Up

A year after the event, the patient continued to do well with significantly reduced and almost negligible symptoms from the incident. She said that her sleep was back to pre-awareness event quality. She still had occasional flashbacks of being awake and unable to move but these flashbacks were short-lived and rare and did not involve the severe anxiety she used to experience. She credited being able to talk about the event and understanding the circumstances as helping her 
with coping post-event. A month before the awareness event she had undergone weight loss surgery and she was happy that because the anxiety from the event was well managed, she had been able to continue with her weight loss goals having lost a total of 140 pounds a year later.

\section{Discussion}

After giving anesthesia to a patient, an unintended return of consciousness after administering general anesthesia is known as Accidental Awareness under General Anesthesia which represents a failure to achieve the primary goal of anesthesia. It is also referred to as intra-operative awareness under general anesthesia. It is a rare complication when a patient gets back their consciousness during the operative or perioperative period when the intention is to have the patient unconscious. While the patient may not experience any pain during this period, they can recall the memories or events spontaneously or prompted that occurred in the operating room at the time. In this particular case, the patient was not in the operating room, but the awareness happened during bedside intubation. Intubation was performed 2 days post-operatively when the patient developed uncontrolled tonsillar hemorrhage after a successful adenotonsillectomy. In this patient, intubation was a necessary emergency procedure to control the tonsillar hemorrhage while a plan was formulated for further surgical intervention. From her recall of events where she was aware but unable to move, we can assume that she had been given an induction agent and a paralytic agent during the intubation but, the induction agent wore off when the effects of the paralytic were still active. This resulted in the inability of the patient to move but was fully aware of her surroundings. The patient's awareness of the events that occurred during intubation was a likely cause of her symptoms suggestive of PSTD where she re-experienced the events and sounds that occurred during the procedure with no success to avoid the experience. She also experienced nightmares and flashbacks which suggest increased arousal following awareness during general anesthesia. Similar cases of awareness during general anesthesia with a PTSD sequela have been reported and documented in literature [4]. Moerman et al. [5] described the experiences of people who had awareness during anesthesia where auditory perceptions were among the most common complaints with feelings of anxiety, panic, and helplessness as reported in our case. Goldmann et al. [6] noted that all patients who had a recall of cardiopulmonary bypass surgery had high postoperative anxiety. The diagnosis of PTSD following awareness during anesthesia is also not uncommon. MacLeod and Maycock [7] published case reports of three patients who developed PSTD following recall of events that occurred during anesthesia.

Although knowledge for the treatment of an experience of awareness during general anesthesia is limited, signs and symptoms of intra-operative awareness reported include a dreamlike experience with varying level of recall of specific events such as: 
- Hearing the conversation in the operation room

- Hearing the noise of tools

- Feeling the pain of surgery

- Not being able to move or feeling weakness and anxiety

Signs that may indicate the intra-operative awareness of a patient includes [8];

- Tachycardia (heart rate over 100 beats per minute)

- Tachypnea (abnormal breathing)

- High blood pressure (hypertension)

- Movement of patient

Remembering past events may be spontaneous or non-spontaneous. After the surgery, a "recall test" can be carried out by the doctors in which they ask the patient whether they remember something during the anesthetic period or not. They may ask the patient if they remember the conversation or sounds of tools. This test is conducted to check the patient's explicit or conscious memory i.e., conscious recollection of past experiences. On the other hand, to check the patient's implicit or unconscious memory, a recognition test is carried out. In this test, a patient is asked to determine whether he or she remembers any word heard during the period of unconsciousness [3].

While the true etiology of this episode's accidental awareness is still unclear, AAGA is known to be more common under emergency surgeries with concurrent hypotension due to hypovolemia or hemorrhage [4]. Formal interviewing by a mental health specialist is ideal to extract maximum information and to provide solace and guidance. A Brice questionnaire can be used as a starting point to get information on the awareness incident [9]. The modified version of the Brice questionnaire over the years has now become the gold standard when studying AAGA. Below is an example of a modified Brice questionnaire [10]:

Modified Brice Questionnaire

1) What is the last thing you remember before your surgery?

2) What is the first thing you remember after waking up?

3) Can you recall anything between under anesthesia and waking up?

4) Did you dream anything during surgery? If so, what is disturbing?

5) What did you find unpleasant about the surgery? Did you have problems going to sleep or waking up?

This patient's event was compounded by the event happening in the middle of the COVID-19 pandemic which caused a limitation of access to specialists as non-urgent office appointments were canceled. Causes of AAGA include drug resistance, suboptimal dosing, or anesthesia machine malfunction [11]. Awareness can be prevented to a great extent by adopting measures like premedication with benzodiazepines, regular checking of the function of anesthesia delivery system, thorough intra-operative monitoring including looking for signs of consciousness, sympathetic overactivity, monitoring of brain electrical activity including EEG (Bispectrality index and Entropy) and evoked brain electrical activity monitoring [8]. 
Follow-up and regular check-ups on the patient should be done if accidental awareness is diagnosed. This is because the effects may not manifest immediately after the unintended awareness under anesthesia. Regular psychological or psychiatric care should be sought as necessary for helping the patient cope and maintain a sense of normalcy after the traumatic event.

\section{Conclusion}

Awareness during general anesthesia is uncommon but can result in long-term disorders such as PTSD. Assessment for awareness should preferably begin in the recovery room with the patient being asked if he or she recalled any experiences during the procedure. Although it may be difficult to perform a similar assessment during bedside emergency procedures that require sedation, from our case report, it seems necessary and reasonable to follow-up patients as closely as possible because emergency procedures are associated with a high risk of awareness. During follow-up, clinicians should ask about nightmares and flashbacks of the event, feelings of fear or terror, sleep disturbance, and relentless memories of the procedure. A patient who acknowledges awareness or who presents with symptoms suggestive of PSTD requires complete evaluation and treatment by a specialized healthcare provider.

\section{Conflicts of Interest}

The authors declare no conflicts of interest regarding the publication of this paper.

\section{References}

[1] Ghoneim, M.M. and Weiskopf, R.B. (2000) Awareness during Anesthesia. Anesthesiology, 92, 597. https://doi.org/10.1097/00000542-200002000-00043

[2] Domino, K.B., Posner, K.L. and Caplan, R.A. (1990) Awareness during Anesthesia: A Closed Claims Analysis. Anaesthesiology, 90, 1053-1061. https://doi.org/10.1097/00000542-199904000-00019

[3] Tasbihgou, S.R., Vogels, M.F. and Absalom, A.R. (2018) Accidental Awareness during General Anesthesia-A Narrative Review. Anesthesia, 73, 112-122. https://doi.org/10.1111/anae.14124

[4] Osterman, J.E. and van der Kolk, B.A. (1998) Awareness during Anesthesia and Posttraumatic Stress Disorder. General Hospital Psychiatry, 20, 274-281. https://doi.org/10.1016/S0163-8343(98)00035-8

[5] Moerman, N., Bonke, B. and Oosting, J. (1993) Awareness and Recall during General Anesthesia: Facts and Feelings. Anesthesiology, 79, 454-464. https://doi.org/10.1097/00000542-199309000-00007

[6] Goldmann, L., Shah, M.V. and Hebden, M.W. (1987) Memory of Cardiac Anesthesia. Psychological Sequelae in Cardiac Patients of Intra-Operative Suggestion and Operating Room Conversation. Anesthesia, 42, 596-603. https://doi.org/10.1111/j.1365-2044.1987.tb03082.x

[7] Macleod, A.D. and Maycock, E. (1992) Awareness during Anesthesia and PostTraumatic Stress Disorder. Anaesthesia and Intensive Care, 20, 378-382. 
https://doi.org/10.1177/0310057X9202000323

[8] AAGA (2014) Accidental Awareness during General Anaesthesia in the United Kingdom and Ireland. Report and Findings.

[9] Brice, D.D., Hetherington, R.R. and Utting, J.E. (1970) A Simple Study of Awareness and Dreaming during Anesthesia. British Journal of Anaesthesia, 42, 535-542. https://doi.org/10.1093/bja/42.6.535

[10] Sandin, R.H., Enlund, G., Samuelsson, P. and Lennmarken, C. (2000) Awareness during Anesthesia: A Prospective Case Study. The Lancet, 355, 707-711.

https://doi.org/10.1016/S0140-6736(99)11010-9

[11] Velankar, P.M. (1994) Awareness during Surgery under Anesthesia (A Case Report). Medical Journal Armed Forces India, 50, 57-58.

https://doi.org/10.1016/S0377-1237(17)31039-0 